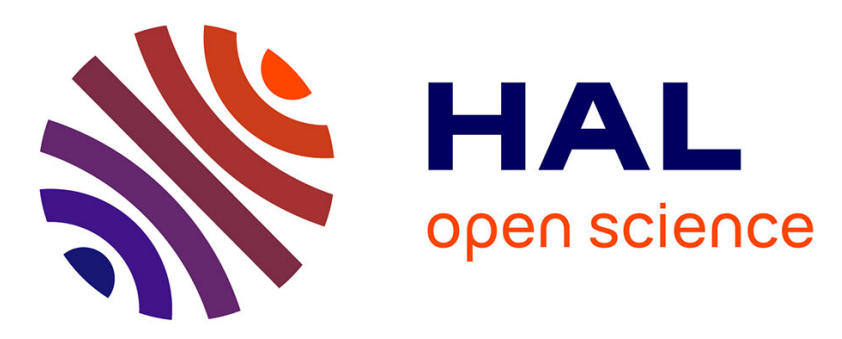

\title{
Quantum-classical model for the surface plasmon enhanced photoemission process at metal surfaces
}

H Jouin, M Raynaud, G Duchateau, G Geoffroy, A Sadou, Patrick Martin

\section{To cite this version:}

H Jouin, M Raynaud, G Duchateau, G Geoffroy, A Sadou, et al.. Quantum-classical model for the surface plasmon enhanced photoemission process at metal surfaces. Physical Review B: Condensed Matter and Materials Physics (1998-2015), 2014, 10.1103/PhysRevB.89.195136 . hal-01327362

\section{HAL Id: hal-01327362 https://hal.science/hal-01327362}

Submitted on 8 Jun 2016

HAL is a multi-disciplinary open access archive for the deposit and dissemination of scientific research documents, whether they are published or not. The documents may come from teaching and research institutions in France or abroad, or from public or private research centers.
L'archive ouverte pluridisciplinaire HAL, est destinée au dépôt et à la diffusion de documents scientifiques de niveau recherche, publiés ou non, émanant des établissements d'enseignement et de recherche français ou étrangers, des laboratoires publics ou privés. 


\title{
Quantum-classical model for the surface plasmon enhanced photoemission process at metal surfaces
}

\author{
H. Jouin, ${ }^{1, *}$ M. Raynaud,${ }^{2}$ G. Duchateau, ${ }^{1}$ G. Geoffroy, ${ }^{1,3}$ A. Sadou,,${ }^{1}$ and P. Martin ${ }^{1}$ \\ ${ }^{1}$ Université Bordeaux, CNRS, CEA, CELIA, UMR 5107, F-33400 Talence, France \\ ${ }^{2}$ CEA/DSM/IRAMIS/LSI, CNRS, Ecole Polytechnique, 91128 Palaiseau Cedex, France \\ ${ }^{3}$ CEA/DSM/IRAMIS/SPAM, 91190 Saclay, France
}

(Received 22 July 2013; revised manuscript received 10 April 2014; published 28 May 2014)

\begin{abstract}
The main purpose of this work is to propose a theoretical description of the surface plasmon polariton stimulated electron emission process at metal surfaces in which the primary electron ejection from the conduction band is treated quantum mechanically in order to go beyond the approximate approaches used up to now to represent this first step. Our theoretical results are well supported by experimental energy spectra obtained for some tens of femtosecond laser pulses impinging on a gold grating target at various intensities in the $\mathrm{GW} / \mathrm{cm}^{2}$ range. The present model which allows us to discuss various features of the surface plasmon photoemission process such as the role of the surface plasmon phase is rather simple and could be applied with slight modifications to study the stimulated photoemission of metallic nanoparticles which has been a subject of growing interest during the past few years.
\end{abstract}

DOI: 10.1103/PhysRevB.89.195136

PACS number(s): 73.20.Mf, 78.47.J-, 79.60.-i

\section{INTRODUCTION}

Short laser pulse excitation of collective electron oscillations in various systems of different scales, such as metal nanoparticles or periodically patterned metal films, has triggered tremendous interest over the last decade. These collective modes called surface plasmons polaritons in the long-wavelength limit [1] relevant for laser excitation will be referred to in what follows only as surface plasmons (SP) for the sake of conciseness. SP laser excitation is attractive as a way to concentrate and channel light and consequently to induce high-electron emission rates [2-9]. As a matter of fact, in metals, SP laser excitation is the sole mechanism able to induce a strong absorption of the incident light (in some cases up to $90 \%$, instead of less than $10 \%$ otherwise) and a large increase of both the energy and the yield of the emitted photoelectrons [10-13]. As a consequence, an intense activity is presently developed to use the SP field properties to enhance nonlinear processes. Many new applications have been proposed such as a new surface plasmon free-electron laser scheme [14], surface plasmon circuits in electronic chips, surface plasmons as trace analyzers and biological probes [15], an attosecond nanoplasmonic microscope [7], and laptop-sized extreme-ultraviolet light sources [8].

Electrons emitted through the SP-enhanced photoelectric effect at metal surfaces are much more energetic than in a conventional photoemission process [16-19]. This phenomenon has been interpreted in terms of ponderomotive acceleration [16] of the electrons since when they are released from the metal, they further experience a vacuum dressed by the inhomogeneous high-frequency longitudinal SP field. Consequently, the total energy of a freed electron traveling outside the metal may be expressed as the sum of the kinetic energy gained after overcoming the metal work function and of its quiver energy $U_{p}=E_{\mathrm{SP}}^{2} / 4 \omega_{0}^{2}$ (or ponderomotive potential) in the oscillating SP field $E_{\mathrm{SP}}\left(\omega_{0}\right.$ being the laser angular frequency). As this field is strongly inhomogeneous,

\footnotetext{
*Corresponding author: jouin@ @elia.u-bordeaux1.fr
}

electrons outside the solid experience a low-frequency nonlinear force which expels them from the (near surface) high intensity field zone towards the low-amplitude region far from the metal. As the SP field amplitude is much higher than the laser field one (up to some hundred times higher), the ponderomotive potential is high even at low laser intensities as those considered in the present work (in the $\mathrm{GW} / \mathrm{cm}^{2}$ range) and the electron is significantly accelerated towards the low-field region. The magnitude of this effect depends both on the pulse duration (the SP lifetime is of the same order as the laser pulse duration [16]) and on the laser intensity since the SP field amplitude is proportional to the laser field one. Moreover, an increment of acceleration might be obtained by the freed electron depending on the phase of the SP field at the instant of its release [16]. This effect which is not of ponderomotive origin has been recently investigated for SP excited by few-cycle and single-cycle laser pulses [20].

Within the last decade, an increasing number of works, either experimental or theoretical, have been dedicated to the SP-stimulated photoelectric effect [16,18,20-24]. In these works, the SP-enhanced emission process has been represented in terms of a two-step mechanism which is also used in the present work. The first step considers an electronic transition from the metallic conduction band towards the metal continuum due to the SP field, while in the second one, these freed electrons move and gain more energy in the inhomogeneous SP field outside the metal. In these works, the second step has been described from a classical point of view and this is also the case here. The first step has been accounted for either through an $I^{n}(t)$ temporal distribution $[18,21]$ [with $I(t)$ the intensity of the laser field and $n$ the number of photons required to eject an electron from the metal] when multiphoton primary ejection is considered or by means of the Fowler-Nordheim equation $[20,22,23]$ to describe tunnel emission. These descriptions are approximate representations for the temporal shape of the primary ejection process and it is not clear how they can account either for the energy distributions or for the angular ones. As the natural frame to describe the first step is quantum mechanics, the ejection probability as a function of the electron energy, as a 
function of the emission angle, and as a function of time are obtained in the present work by means of a perturbative method called "jellium-Volkov" [25]. In that way, these momentum, angular, and temporal distributions can be used to define unambiguously the initial conditions of the second (classical) step. However, we will show that particular attention must be paid to the connection between both steps. Our model allows us to obtain stimulated photoemission energy spectra which are in good agreement with measurements for rather short laser pulses (some tens of fs) impinging on a gold grating at various intensities in the range $10^{9}-10^{10} \mathrm{~W} / \mathrm{cm}^{2}$.

This paper is organized as follows: The theoretical model is first detailed in Sec. II while the framework of our experiment is presented in Sec. III together with comparisons between measured and calculated energy spectra. Finally, conclusions are given in Sec. IV. Atomic units are used throughout this paper unless otherwise stated.

\section{MODEL}

\section{A. Description of the SP field}

We follow the approach of Refs. [20,22,26] in which an analytical expression is used to describe the SP field. More refined solutions of Maxwell's equations for the SP field have been obtained in Refs. [18,21] by means of a finite-difference time-domain numerical approach. Nevertheless, it would be very cumbersome to combine the quantum-mechanical results of the present calculations with the solutions of such a numerical approach to describe the SP field. As our aim is to build a model as simple as possible without losing the main physical features, we have considered for the two components of the SP electric field outside the metal $\left(\mathrm{SP}_{e}: z \geqslant 0\right)$

$$
\begin{aligned}
E_{x}^{\mathrm{SP}_{e}}(x, z, t) & =\beta \eta E_{0} \mathcal{F}(x, t) \exp \left(-z / \delta_{e}\right) \sin \left(\omega_{0} t-k_{\mathrm{SP}} x+\phi_{0}\right), \\
E_{z}^{\mathrm{SP}_{e}}(x, z, t) & =\eta E_{0} \mathcal{F}(x, t) \exp \left(-z / \delta_{e}\right) \cos \left(\omega_{0} t-k_{\mathrm{SP}} x+\phi_{0}\right), \\
\mathcal{F}(x, t) & =\cos ^{2}\left[\pi\left(t-\frac{k_{\mathrm{SP}}}{\omega_{0}} x\right) / \tau\right] \Pi\left(t-\frac{k_{\mathrm{SP}}}{\omega_{0}} x\right),
\end{aligned}
$$

with $x$ and $z$ the coordinates parallel and perpendicular to the surface plane, respectively. $E_{0}, \omega_{0}, \phi_{0}$, and $\tau$ are the amplitude of the incident laser field, the carrier frequency, the phase, and the SP duration, respectively. $\mathcal{F}(x, t)$ is the envelope function in which $\Pi\left(u \equiv t-\frac{k_{\mathrm{SP}}}{\omega_{0}} x\right)=\Theta(u+\tau / 2)-\Theta(u-\tau / 2)$ is the "window" function with $\Theta$ the Heaviside step function. We have chosen to use a $\cos ^{2}$ envelope form instead of the more usual Gaussian form which is generally considered to fit experimental laser pulses envelopes, since the $\cos ^{2}$ form enables us to perform analytically a part of the quantummechanical calculations. It is worth noting that with this $\cos ^{2}$ form, the SP full width at half maximum is $\tau / 2$. Since the experimental spectra presented in Sec. III have been obtained in the case of 58 fs full width at half maximum Gaussian laser pulses, all the calculations reported below have been performed for $\tau=116$ fs (=4796 a.u.). Indeed, autocorrelation measurements [16] have shown that the SP lifetime is of the same order as the laser pulse duration; we assume in this work that they are identical.
The SP field components inside the metal $\left(\mathrm{SP}_{i}: z \leqslant 0\right)$ have the same forms as those given in Eq. (1) except for the decay length $\delta_{e}$ that becomes $\delta_{i}$ for the internal part. These parameters $\delta_{e}$ and $\delta_{i}$, as well as $k_{\mathrm{SP}}$ (the SP wave vector component parallel to the surface) and $\beta$ (the ratio between the amplitude of the SP field component parallel to the surface and the perpendicular one) are characteristics of the SP. Their expressions as a function of the metal dielectric function $\epsilon\left(\omega_{0}\right)$ are obtained by consideration of the continuity relations of the fields inside and outside the metal [27], and can be expressed as

$$
\begin{aligned}
k_{\mathrm{SP}} & =\frac{2 \pi}{\lambda_{0}} \sqrt{\frac{\epsilon\left(\omega_{0}\right)}{\epsilon\left(\omega_{0}\right)+1}}, \quad \delta_{e}=\frac{\lambda_{0}}{2 \pi} \sqrt{-\left[\epsilon\left(\omega_{0}\right)+1\right]}, \\
\delta_{i} & =\delta_{e} /\left|\epsilon\left(\omega_{0}\right)\right|, \quad \beta=\left|\epsilon\left(\omega_{0}\right)\right|^{-1 / 2} .
\end{aligned}
$$

In the case considered here, application of these formulas with $\epsilon\left(\omega_{0}=0.057\right.$ a.u. $)=-22.7$ [28] and $\lambda_{0}=800 \mathrm{~nm}$ (the laser wavelength) leads to $\delta_{e}=593.8 \mathrm{~nm}$ (三11221 a.u.), $\delta_{i}=26.1 \mathrm{~nm}$ (三494 a.u.), and $\beta=0.21$. One can note that consideration of the $\epsilon\left(\omega_{0}\right)$ value $(\simeq-24)$ obtained from Ref. [29] leads to slightly different values for these parameters (respectively, $\delta_{e}=610.6 \mathrm{~nm}, \delta_{i}=26.5 \mathrm{~nm}$, and $\beta=0.20$ ); we have checked that such variations do not modify the final results presented in Sec. III.

Finally, to obtain the enhancement factor of the SP field with respect to the laser one $\left(\eta=E_{\mathrm{SP}} / E_{0}\right)$, we adjust this value in order to obtain the best agreement between the calculated energy spectra and the experimental ones. This is the only free parameter of our model.

\section{B. Primary ejection of electrons: Quantum calculations}

In the low laser intensity regime considered here, the measured current density scales as $I_{L}^{4}$ (with $I_{L}$ the maximum laser intensity) [16] which is the signature of an electronic emission driven by a four-photon absorption. In that way the thermally assisted photoemission process [30,31] in which electrons in the high-energy tail of the transient thermal distribution play a significant role can be disregarded in the present case. Thus, in order to describe the primary step of the SP stimulated emission process, we have chosen to use a perturbative quantum-mechanical method called "jellium-Volkov" (JV) [25] which is less time consuming than nonperturbative approaches such as the time-dependent Schrödinger equation resolution [25,32] or more refined timedependent density functional theory methods. In this approach, we consider only the time-dependent part of the SP field component perpendicular to the surface which reads

$$
\mathcal{E}_{z}^{\mathrm{SP}}(t)=\eta E_{0} \cos ^{2}\left(\frac{\pi t}{\tau}\right) \cos \left(\omega_{0} t+\phi_{0}\right) \Pi(t) .
$$

In other words, the SP field component parallel to the surface has been neglected in this quantum part of the model. This approximation, which is justified here since the normal component of the SP electric field is 5 times greater than the parallel one, amounts to considering that the electronic momentum component parallel to the surface is conserved during this primary step. 
Within the JV approach, the transition amplitude for the ejection of an electron from the metal due to the $\mathcal{E}_{z}^{\mathrm{SP}}(t)$ field is written as

$$
T_{f i}(t)=-i \int_{-\infty}^{t} d t\left\langle\Psi_{k_{f}}(z, t)|\mathcal{V}(z, t)| \Phi_{k_{i}}(z)\right\rangle,
$$

where $\mathcal{V}(z, t)=z \mathcal{E}_{z}^{\mathrm{SP}}(t)$ is the interaction potential corresponding to the field of Eq. (3) while $\Phi_{k_{i}}(z)$ is the initial unperturbed wave function representing an electron in the metal conduction band with the normal momentum component $k_{i}$ and $\Psi_{k_{f}}(z, t)$ is the final perturbed wave function representing an ejected electron in both the metal and the SP potentials.

The metallic electronic structure is described through the jellium model and the metal potential is written $V_{M}(z)=$ $-V_{c} \Theta(-z)$ where $V_{c}=E_{F}+W$ with $E_{F}=5.5 \mathrm{eV}$ the Fermi energy and $W=5.1 \mathrm{eV}$ the work function [33]. Under this framework, one obtains analytical expressions for both the initial $\left[\Phi_{k_{i}}(z)\right]$ and final $\left[\Phi_{k_{f}}(z)\right]$ unperturbed states by solving the corresponding 1D stationary Schrödinger equation [34]. The so-called jellium-Volkov state [25] representing the final wave function $\Psi_{k_{f}}(z, t)$ reads

$$
\Psi_{k_{f}}(z, t)=\Phi_{k_{f}}(z) \exp \left(i \frac{z}{c} \mathcal{A}(t)\right),
$$

where $\mathcal{A}(t)=-c \int_{-\tau / 2}^{t} \mathcal{E}_{z}^{\mathrm{SP}}\left(t^{\prime}\right) d t^{\prime}$ is the vector potential and $c$ the speed of light in vacuum. Such an approach based on the dressing of unperturbed states by the Volkov phase has been shown to properly describe multiphoton absorption in various systems [25,35-37]. In the present work, the ponderomotive energy term appearing in the Volkov phase $[25,37]$ has been removed since this allows the elimination of spurious behaviors which have been shown to appear [38] in the above-threshold ionization (ATI) process for atoms and also to speed up the calculations. Due to the analytical forms of both the initial and final unperturbed states related to the step potential description of the metallic edge (which amounts to neglecting image effects on the initial and final unperturbed wave functions), the integral over electron coordinates (over the $z$ coordinate in the present case) resulting from Eq. (4) can be written in a closed form. We perform the final time integral of Eq. (4) by means of a Simpson quadrature.

As previously stated, in Eq. (3), only the time-dependent part of the normal SP field component is considered. However test calculations taking into account the inhomogeneous part of the normal SP field component, i.e., $\mathcal{E}_{z}^{\mathrm{SP}}(t) \times$ $\left[\exp \left(-z / \delta_{e}\right) \Theta(z)+\exp \left(z / \delta_{i}\right) \Theta(-z)\right]$, and the corresponding scalar potential have been performed in order to check the accuracy of the approximate field description of Eq. (3). The results are very close to those presented in what follows since the length scale involved in the primary ejection is very small with respect to the SP decay lengths $\left(\delta_{e, i}\right)$. However, it can be useful to keep the decay term inside the solid to avoid numerical problems since after spatial integration, the remaining time integral contains the following term: $\left[k_{i z}-k_{f z}-\mathcal{A}(t)+i \delta_{i}\right]^{-2}$. In the case of $\delta_{i}=0$, a diverging behavior can appear during the time-integration procedure for $\mathcal{A}(t)=k_{i z}-k_{f z}$.

With the transition amplitude $T_{f i}$ calculated as indicated above, we obtain the differential ejection probability as a function of time for a fixed energy $\varepsilon_{f}$ in the metal continuum and for a given angle of emission $\theta_{e}$ with respect to the surface plane (corresponding to the elementary solid angle $d \Omega_{f}$ ). This distribution that will be referred to (for simplicity) in what follows as $D\left(\theta_{e}, \varepsilon_{f}, t\right)$ is obtained by means of the expression

$$
\begin{aligned}
D\left(\theta_{e}, \varepsilon_{f}, t\right) & =\frac{\partial^{2} P}{\partial \varepsilon_{f} \partial \Omega_{f}} \\
& =\frac{k_{f}^{\prime} k_{f z}^{\prime}}{k_{f z}} \int d \vec{k}_{i} \Theta\left(k_{F}-k_{i}\right) \rho\left(k_{i}\right)\left|T_{f i}(t)\right|^{2}
\end{aligned}
$$

and provides the instants in which the electrons are emitted as well as the energy and angular distribution for primary ejection at the end of the SP duration (which ranges here between $-\tau / 2$ and $+\tau / 2)$ through $\mathcal{D}\left(\theta_{e}, \varepsilon_{f}\right)=D\left(\theta_{e}, \varepsilon_{f}, t=+\tau / 2\right)$. In Eq. (6), $\varepsilon_{f}=k_{f}^{\prime 2} / 2$ is the final electron energy measured with respect to the ionization threshold and $k_{f}^{\prime}$ is the modulus of the corresponding wave vector whose $z$ component is given by $k_{f z}^{\prime}=\left(k_{f z}^{2}-2 V_{c}\right)^{1 / 2}$ (with $k_{f z}$ measured with respect to the bottom of the conduction band). $\Theta\left(k_{F}-k_{i}\right)$ in Eq. (6) stands for the zero-temperature limit of the Fermi-Dirac distribution while $\rho\left(k_{i}\right)$ is the density of initial states which in the free electron gas description considered here reads [39] $\rho\left(k_{i}\right)=\left(3 k_{i}\right) / k_{F}^{3}$ (with $k_{F}=\sqrt{2 E_{F}}$ the Fermi wave vector modulus). Furthermore, the constraint $0 \leqslant k_{i} \leqslant k_{F}$ combined with energy conservation makes the integration range over the $k_{i z}$ momentum component to be limited by the values $k_{i z}^{\min }=0$ (in all cases) and $k_{i z}^{\max }$ which depends on both the final energy $\varepsilon_{f}$ and the ejection angle $\theta_{e}$ through

$$
k_{i z}^{\max }=\sqrt{2\left(E_{F}-\varepsilon_{f} \cos ^{2} \theta_{e}\right)} .
$$

We present in Fig. 1 energy and angle distributions in (a) and time distribution in (b) obtained for the primary electron emission by means of the JV approach for the following parameters (that will be used in one of the comparisons to experiments presented in Sec. III): $\tau=116 \mathrm{fs}, \xi_{0}^{\mathrm{SP}}=\eta E_{0}=0.031$ a.u., and $\phi_{0}=0$. In Fig. 1(a) are displayed the normalized energy distributions obtained at the end of the SP duration (i.e., at $t=+\tau / 2$ ) for three emission angles: $\theta_{e}=90^{\circ}, \theta_{e}=50^{\circ}$, and $\theta_{e}=10^{\circ}$. The maximum of the distribution corresponding to $\theta_{e}=90^{\circ}$ (i.e., for $\varepsilon_{f} \simeq 4 \mathrm{eV}$ ) has been normalized to unity and the other distributions related to $\theta_{e}=50^{\circ}$ and $\theta_{e}=10^{\circ}$ have been normalized accordingly. As can be observed in Fig. 1(a), the energy distributions exhibit various peaks corresponding to the absorption of several photons. The first peak close to $1.1 \mathrm{eV}$ corresponds to the minimum number of photons required to remove an electron from the Fermi level, i.e., $n=4$, and it is a simple matter to verify that its energy location correctly accounts for energy conservation $\left(\varepsilon_{n}=n \times \hbar \omega_{0}-W\right)$. The next peaks which correspond to the absorption of further photons for an electron in the metal continuum (ATI) are then regularly shifted of $\hbar \omega_{0}=1.55 \mathrm{eV}$. For normal emission with respect to the surface $\left(\theta_{e}=90^{\circ}\right)$, it can be observed that the energy distribution mainly ranges within 0 and $8 \mathrm{eV}$. In agreement with Eq. (7), this energy range as well as the number of emitted electrons get reduced with decreasing ejection angles. This behavior which means that normal emission is dominant (although emission at nonperpendicular angles is not negligible) has been already analyzed in Ref. [25] and is 


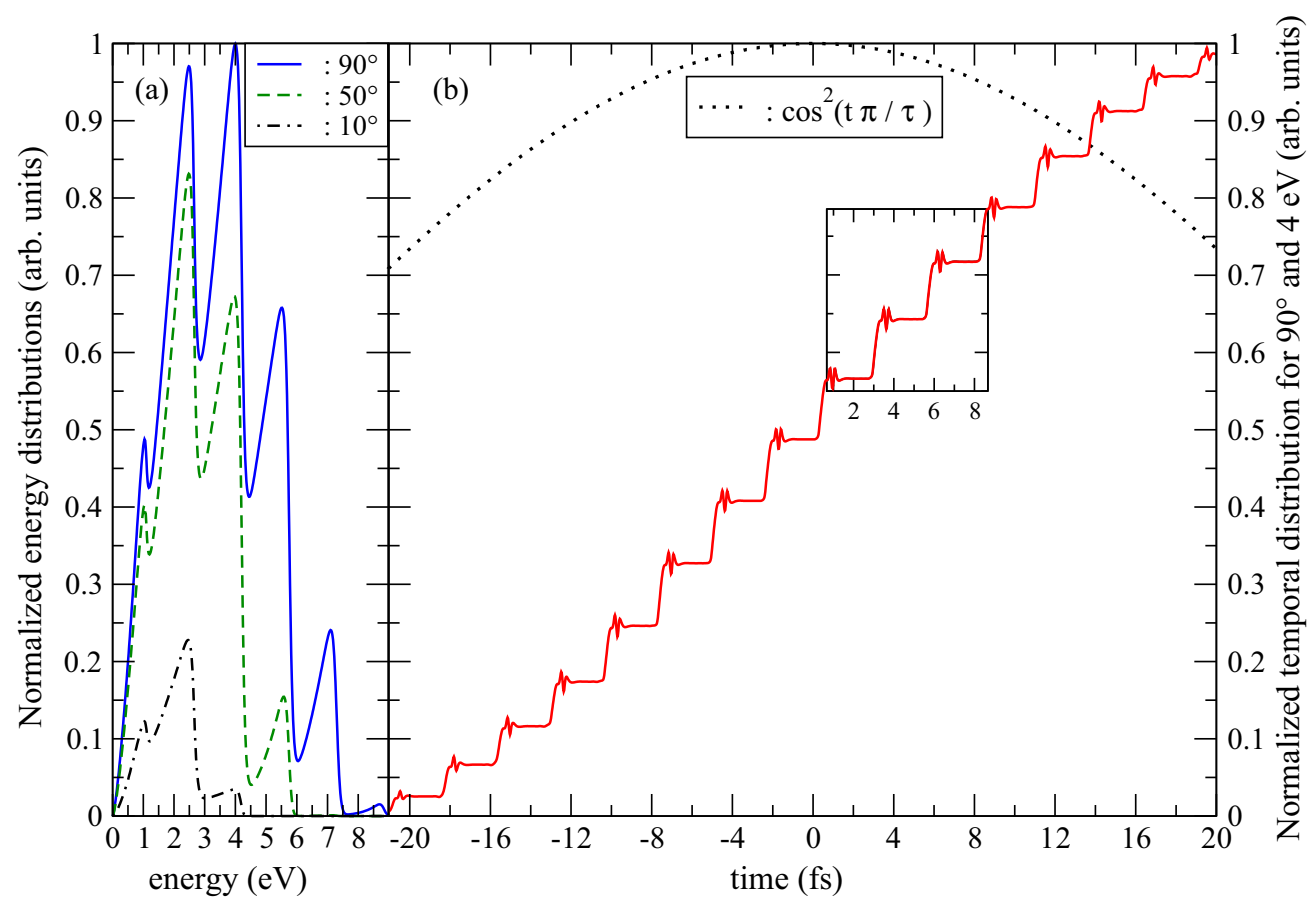

FIG. 1. (Color online) Results of jellium-Volkov calculations related to primary electron emission for $\tau=116 \mathrm{fs}, \xi_{0}^{\mathrm{SP}}=\eta E_{0}=0.031$ a.u., and $\phi_{0}=0$. (a) Normalized spectra $\mathcal{D}\left(\theta_{e}, \varepsilon_{f}\right)$ of primary ejected electrons as a function of energy (in eV) for three emission angles: $\theta_{e}=90^{\circ}$ : full blue line, $\theta_{e}=50^{\circ}$ : dashed green line, $\theta_{e}=10^{\circ}$ : dot-dashed black line. (b) Full red line: evolution of the normalized ionization probability $D\left(\theta_{e}, \varepsilon_{f}, t\right)$ as a function of time (in fs) for $\theta_{e}=90^{\circ}$ [i.e., corresponding to the full blue line energy spectrum of panel (a)] and for an emission energy of $\varepsilon_{f}=4 \mathrm{eV}$; dotted black line: SP temporal envelope shape [i.e., $\cos ^{2}(\pi t / \tau)$ ]. The inset corresponds to three periods that will be used in what follows to analyze the temporal emission characteristics (see text).

consistent with the fact that we have considered in the present quantum calculations only the perpendicular component of the SP field since it is stronger than the parallel one. Calculations (not reported) for other values of the phase $\phi_{0}$ [than the value $\phi_{0}=0$ of Fig. 1(a)] show that the energy distributions do not present any variation with this parameter for the (rather long) SP duration considered here. However, we have observed that this is not the case for few-cycle SP durations for which energy distributions show a noticeable dependence on $\phi_{0}$. Figure 1(b) displays the evolution of the normalized ionization probability as a function of time for $\theta_{e}=90^{\circ}$ and for an emission energy of $\varepsilon_{f}=4 \mathrm{eV}$ which corresponds to the maximum probability of ejection for this emission angle [see Fig. 1(a)]. First, one can notice that the emission takes place in between the third central part of the SP envelope [this envelope is depicted by the dotted line in Fig. 1(b)]. This distribution increases step by step with the periodicity of the SP field which is also the laser one; i.e., $T_{0}=2 \pi / \omega_{0} \simeq 2.67 \mathrm{fs}$.

In order to perform a detailed analysis of this temporal distribution pattern which is of great importance for our purpose, we will focus now on three periods between $0.67 \mathrm{fs}$ and $8.68 \mathrm{fs}$ which have been selected in the inset of Fig. 1(b). At the beginning of the period, one can observe small structures which are followed by a central region without any emission and finally, at the end of the period, a rapid increase of the emission. This behavior which is repeated for each period can be more easily analyzed if one considers the time derivative of the temporal distribution $d D\left(\theta_{e}, \varepsilon_{f}, t\right) / d t$. This quantity is reported (full line) in Fig. 2(a) for the three periods selected previously together with the shape of the force (dashed line), i.e., $-\mathcal{E}_{z}^{\mathrm{SP}}(t)$. Within the first quarter of a cycle, one can see that the small structures of $D\left(\theta_{e}, \varepsilon_{f}, t\right)$ in Fig. 1(b) correspond to a series of five zones related to emission and reabsorption which results in a weak net emission. In that first quarter of a period that we call the "secondary emission region," the force [dashed line in Fig. 2(a)] is increasing in the vacuum direction. Further, neither emission nor absorption occurs during the half central part of the cycle; and then within the latter quarter of period that we call the "main emission region" one observes a broad emission peak related to the strong increase of $D\left(\theta_{e}, \varepsilon_{f}, t\right)$ in Fig. 1(b) and corresponding to a force directed towards the metal with decreasing amplitude. These characteristics are repeated for each cycle with a modulation related to the SP field strength [this latter behavior will appear more clearly in Fig. 3(a)]. Furthermore, we have reported in Fig. 2(b) the time derivative of $D\left(\theta_{e}=90^{\circ}, \varepsilon_{f}=4 \mathrm{eV}, t\right)$ obtained for the phase value $\phi_{0}=\pi$ within the same time interval as in Fig. 2(a). As expected, the shape of the force is time shifted with respect to the case $\phi_{0}=0$ of Fig. 2(a) and also the emission features previously described. The main emission region still corresponds to the last quarter of a period in which the force presents a decreasing amplitude and is directed towards the solid while the secondary emission zone is related again to the first quarter of cycle in which the force is directed towards the vacuum with increasing values.

The temporal emission pattern provided by the jelliumVolkov approach is a complicated function of both the SP field and the associated vector potential whose phase difference is $\pi / 2$ with respect to the field. One can find in Ref. [40] the JV transition amplitude written explicitly as a function of 


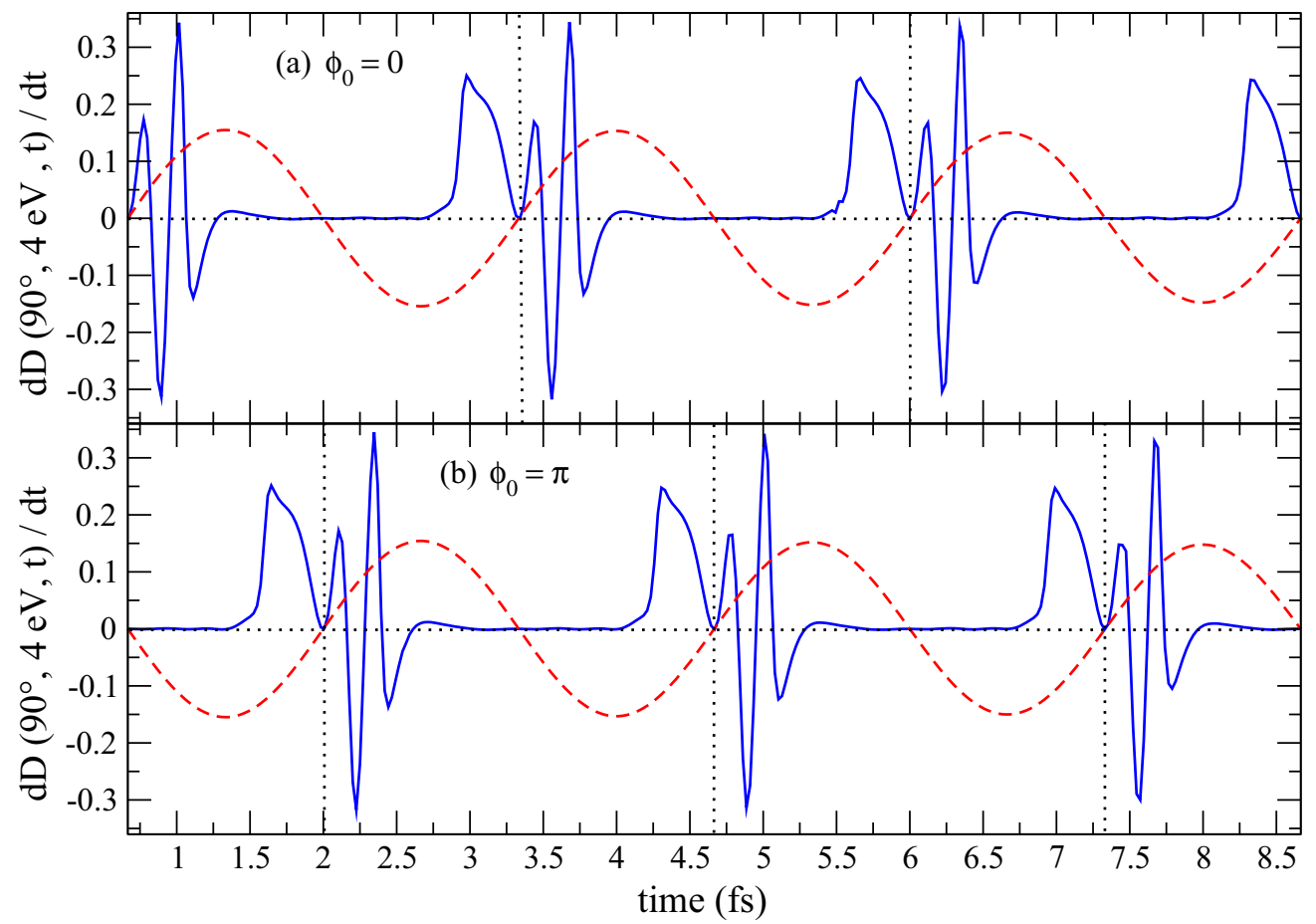

FIG. 2. (Color online) (a) Full blue line: time derivative of the temporal distribution $D\left(\theta_{e}=90^{\circ}, \varepsilon_{f}=4 \mathrm{eV}, t\right)$ as a function of time (in fs) for the three cycles in the inset of Fig. 1(b) corresponding to $\phi_{0}=0$. Dashed red line: $-\mathcal{E}_{z}^{\mathrm{SP}}(t)$; the vertical dotted lines indicate the cycle limits. (b) The same for $\phi_{0}=\pi$.

both $\mathcal{E}_{z}^{\mathrm{SP}}(t)$ and $\mathcal{A}(t)$ in a simplified case. If the Volkov phase which contains $\mathcal{A}(t)$ [see Eq. (5)] is removed, one obtains a first Born approximation type method. In that case, the emission rate would be located around the maxima of the pulling force $\left[-\mathcal{E}_{z}^{\mathrm{SP}}(t)\right]$ as is also obtained [41] by consideration of a Keldysh type tunneling rate in which the instantaneous electric field is considered.

It follows that temporal emission at the metal surface consists in series of bunches of electrons emitted at the SP (or laser) frequency within the first and the last quarter of cycle. These characteristics have two important consequences. First, as we shall see later, the final spectra are almost independent on the value of $\phi_{0}$ for surface waves composed of some tens of periods (or more) as the one considered here (42 cycles). This is not the case for few-cycle surface waves. Moreover, the two temporal zones within the cycle in which the electrons are emitted are favorable for the further acceleration of these latter outside the solid. This point is very important to obtain theoretical spectra whose shape is compatible with their experimental counterpart. To conclude this part, it is worth noting that the temporal emission structures analyzed above do not vary noticeably for different kinetic energies $\varepsilon_{f}$ and different ejection angles.

\section{Motion of the freed electrons in the SP field: Classical calculations}

In this second step, the motion of the freed electrons whose initial kinetic energy, angular distributions, and temporal emission regions are obtained by means of the previous quantum calculations is described from the classical point of view by integration of Newton's second law, which reads in the present case

$$
\begin{aligned}
d x / d t & =p_{x}, \quad d z / d t=p_{z}, \\
d p_{x} / d t & =-E_{x}^{\mathrm{SP}_{e}}(x, z, t)+p_{z} B_{y}^{\mathrm{SP}_{e}}(x, z, t), \\
d p_{z} / d t & =-E_{z}^{\mathrm{SP}_{e}}(x, z, t)-p_{x} B_{y}^{\mathrm{SP}_{e}}(x, z, t),
\end{aligned}
$$

where $x\left(p_{x}\right)$ and $z\left(p_{z}\right)$ are the instantaneous electron vector position (momentum) components parallel and perpendicular to the surface plane, respectively. $E_{x}^{\mathrm{SP}_{e}}$ and $E_{z}^{\mathrm{SP}_{e}}$ are the corresponding SP electric field components outside the metal given in Eq. (1). In Eq. (8), $B_{y}^{\mathrm{SP}_{e}}(x, z, t)$ stands for the magnetic field component along the axis perpendicular to the incidence plane which is the only component of the magnetic field associated with the SP. The explicit lengthy expression of $B_{y}^{\mathrm{SP}_{e}}(x, z, t)$ is obtained from Maxwell-Faraday's law by consideration of the electric field components of Eq. (1). The set of first-order coupled differential equations of Eq. (8) is integrated by means of the adaptative stepsize Cash-Karp Runge-Kutta algorithm provided in Ref. [42]. For each electron (or set of electrons) considered in the statistical approach described below, this set of differential equations is integrated until the values of both the kinetic energy $\left[\left(p_{x}^{2}+p_{z}^{2}\right) / 2\right]$ and the outgoing angle with respect to the surface plane $\left[\arctan \left(p_{z} / p_{x}\right)\right]$ have converged to a relative accuracy better than $10^{-12}$.

To start the integration of Eq. (8), we need to know (for a fixed value of the SP phase $\phi_{0}$ ) the number of electrons $\mathcal{N}_{e}\left(x_{0}, z_{0}, p_{x 0}, p_{z 0}, t_{0}\right)$ emitted as a function of the initial momenta $p_{x 0}$ and $p_{z 0}$ of the instant of emission $t_{0}$ and of the initial positions $x_{0}$ and $z_{0}$. Since in the present work we do not calculate absolute theoretical spectra, an arbitrary number of 


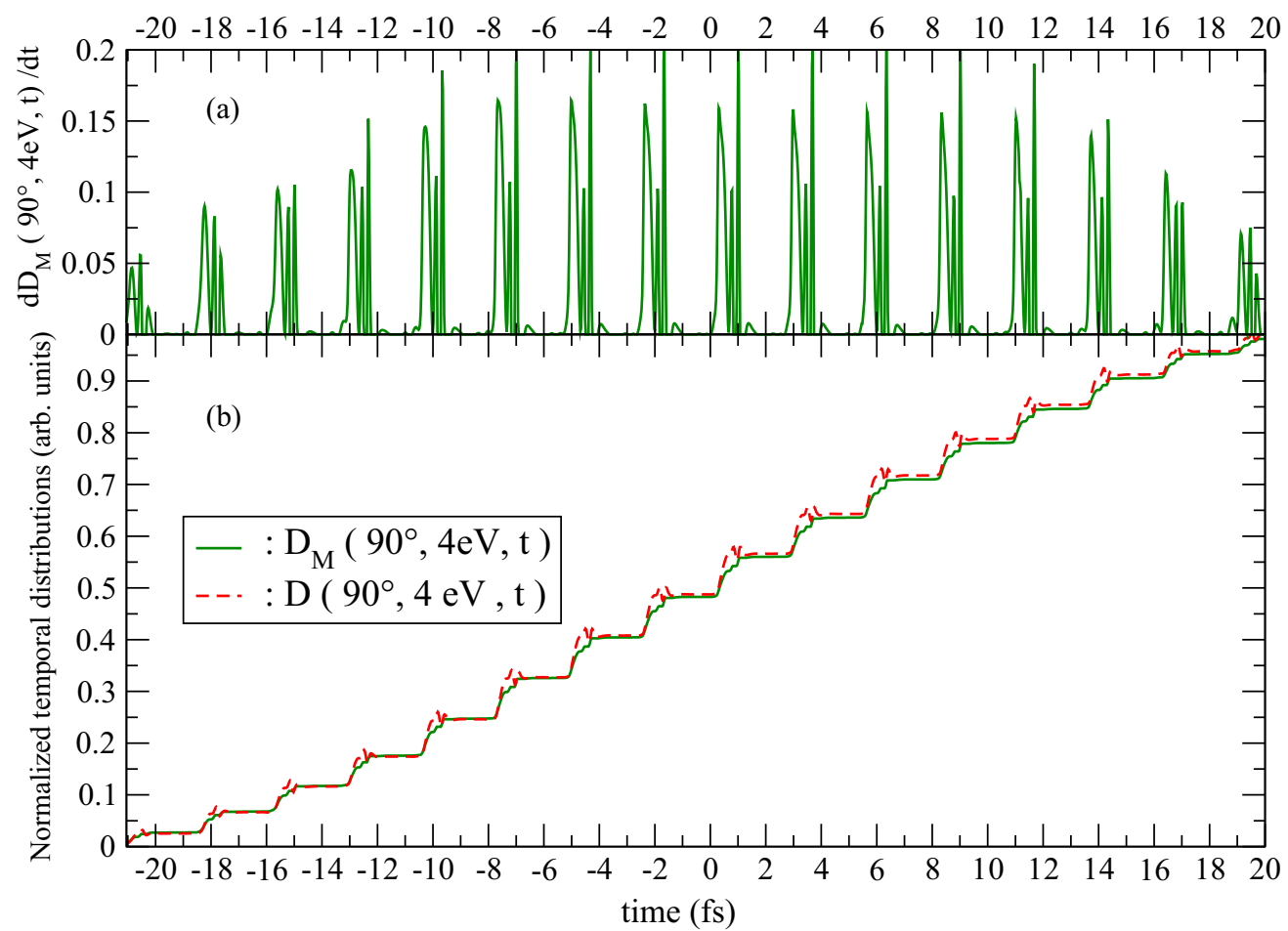

FIG. 3. (Color online) (a) Modified (see text) time derivative $d D_{M}\left(\theta_{e}=90^{\circ}, \varepsilon_{f}=4 \mathrm{eV}, t\right) / d t$ of the temporal distribution as a function of time (in fs) for the third central part of the SP duration. (b) Full green line: modified (see text) temporal distribution of ejected electrons $D_{M}\left(\theta_{e}=90^{\circ}, \varepsilon_{f}=4 \mathrm{eV}, t\right)$. Dashed red line: original temporal distribution of ejected electrons $D\left(\theta_{e}=90^{\circ}, \varepsilon_{f}=4 \mathrm{eV}, t\right)$ already reported in Fig. 1(b). These results correspond to $\phi_{0}=0$ and the time scale is the same as the one of Fig. 1(b).

electrons (typically $\sim 2 \times 10^{6}$ for a good statistics) is allocated to the area of the energy spectrum corresponding to $\theta_{e}=90^{\circ}$. For the other energy spectra related to $\theta_{e} \neq 90^{\circ}$, the number of electrons is obtained accordingly in such a way that the electron number is decreasing for decreasing values of $\theta_{e}$ [see Fig. 1(a)]. The final energy spectra reported in Sec. III have been obtained by consideration of 9 primary energy spectra corresponding to values of $\theta_{e}$ in the range $\left[0,90^{\circ}\right]$. Converged final angular spectra, as the one reported in Fig. 7 , require much more primary energy spectra (around $45 \theta_{e}$ values). These primary spectra are discretized in elementary areas corresponding to momentum intervals $\left[p_{f_{j}}, p_{f_{j+1}}\right]$ (with $p_{f}=\sqrt{2 \varepsilon_{f}}$ ) which provide the number $n_{e}\left(p_{x 0_{j}}, p_{z 0_{j}}\right)$ of electrons emitted for fixed values of $p_{x 0_{j}}=p_{f_{j+1 / 2}} \cos \theta_{e}$ and $p_{z 0_{j}}=p_{f_{j+1 / 2}} \sin \theta_{e}$ where $p_{f_{j+1 / 2}}$ is the midpoint of the momentum interval. Then, from the temporal point of view, these $n_{e}\left(p_{x 0_{j}}, p_{z 0_{j}}\right)$ electrons are emitted according to temporal distributions like the one reported in Fig. 1(b).

In order to solve the problem related to the re-absorption regions, we use a procedure (detailed in the Appendix) which amounts to eliminating the negative values of $d D / d t$ (see Fig. 2) from the temporal distribution and to normalizing the remaining positive part to obtain a final probability (at $t=+\tau / 2$ ) identical to the original one. We display in Fig. 3(a) this modified time derivative of the temporal distribution $\left(d D_{M} / d t\right)$ for the third central part of the SP duration while in Fig. 3(b), one can see that the corresponding modified temporal distribution $\left[D_{M}(t)=\int\left(d D_{M} / d t\right) d t\right]$ is very close to the $D(t)$ one originally obtained from the JV calculation. Hence, the $n_{e}\left(p_{x 0_{j}}, p_{z 0_{j}}\right)$ electrons obtained through the discretization of the energy spectra are distributed according to $d D_{M} / d t$ which leads to $N_{e}\left(p_{x 0_{j}}, p_{z 0_{j}}, t_{0_{k}}\right)$, with

$$
\int_{-\tau / 2}^{+\tau / 2} N_{e}\left(p_{x 0_{j}}, p_{z 0_{j}}, t_{0}\right) d t_{0}=n_{e}\left(p_{x 0_{j}}, p_{z 0_{j}}\right) .
$$

Then, spatially these $N_{e}\left(p_{x 0_{j}}, p_{z 0_{j}}, t_{0_{k}}\right)$ electrons are placed around the peak of the SP pulse in the $x$ direction (within the third central part). And finally, we have used $z_{0}=0$ in all cases since we have observed that our final results do not vary significantly with the initial location of the electrons in the $z$ direction due to the very slow decay of the SP field in that direction.

\section{RESULTS AND DISCUSSION}

\section{A. Experiments}

The experiment has been carried out by using an amplified mode-locked Ti:sapphire laser able to provide $85 \mathrm{~mJ}$ laser pulses at $800 \mathrm{~nm}, 58 \mathrm{fs}$, and $10 \mathrm{~Hz}$ repetition rate (LUCA laser facility in Saclay). As is now well known [27], light may excite SP on a modulated metal surface, the modulation providing the extra momentum necessary to satisfy momentum conservation in photon-plasmon coupling. Indeed, the SP wave vector component parallel to the surface $\left(k_{\mathrm{SP}}\right)$ may be expressed [27] either as a function of the light wave vector $k_{0}=\omega_{0} / c$ and the grating parameter $a$ or as a function of the metal dielectric 
function $\epsilon\left(\omega_{0}\right)$ as

$$
k_{\mathrm{SP}}=\frac{\omega_{0}}{c} \sin \theta+m \frac{2 \pi}{a} \equiv \frac{\omega_{0}}{c} \sqrt{\frac{\epsilon\left(\omega_{0}\right)}{\epsilon\left(\omega_{0}\right)+1}},
$$

where $\theta$ is the angle of incidence of the laser beam (with respect to the surface normal) in $p$ polarization (the only possible polarization for SP excitation by light) and $m$ an integer. From Eq. (10), it is a simple matter to obtain the resonant angles as $\sin \theta_{m}=\left\{\epsilon\left(\omega_{0}\right) /\left[\epsilon\left(\omega_{0}\right)+1\right]\right\}^{1 / 2}-m\left(\lambda_{0} / a\right)$ with $\lambda_{0}$ the laser wavelength. In our experiment, we use a commercial, sinusoidal, holographically registered gold grating sample with 150 grooves per millimeter covered by a 200-nm-thick vacuum-deposited Au film. Application of the previous formula with $\epsilon\left(\omega_{0}=0.057\right.$ a.u. $)=-22.7$ [28], $\lambda_{0}=800 \mathrm{~nm}$, and $a=6667 \mathrm{~nm}$ corresponding to 150 grooves per millimeter leads to the value of $\theta_{1}=64.5^{\circ}$ for the resonant angle corresponding to $m=1$, in good agreement with the experimental measurement of $65^{\circ}$.

The gold sample is held in a magnetically shielded, highvacuum $\left(10^{-8}\right.$ mbar $)$ chamber and can be rotated on its vertical axe to change the laser incidence. The laser beam, the incidence plane of which is perpendicular to the grating grooves, is impinging onto the target without focalization. The laser intensity is deduced from a calorimetric measurement of the pulse energy (with shot-to-shot fluctuations of 5\%) and from the autocorrelation determination of the pulse duration (with a $10 \%$ uncertainty). The electron energy distribution is analyzed with a time-of-flight magnetically shielded spectrometer. To avoid space charge effects a very small extraction potential is applied $(3 \mathrm{~V})$. The energy resolution ranges from $\sim 0.1 \mathrm{eV}$ for energies of some $\mathrm{eV}$ to $\sim 1 \mathrm{eV}$ for energies of some tens of eV. All the data reported below have been obtained for an experimental incidence angle of $65^{\circ}$ with respect to the surface normal, which corresponds to the maximum number of emitted electrons. We have also checked that for this angle of incidence, the electron emission exhibits a strong dependence on laser polarization: the electronic current is much stronger in the $p$-polarization case (which is the signature for SP excitation) than in the $s$ case. To complete the emission analysis, laser absorption measurements have been also performed for $58 \mathrm{fs}$ laser pulse duration and $10^{9} \mathrm{~W} / \mathrm{cm}^{2}$ laser intensity. Within the conditions for SP excitation (i.e., $65^{\circ}$ incidence angle and $p$ polarization), we get $34 \%$ of the laser light absorbed while in the $s$-polarization case (and the same incidence angle) we obtained $20 \%$.

Investigation of electron emission as a function of the laser intensity has been performed in the range $10^{9}$ to $10^{10} \mathrm{~W} / \mathrm{cm}^{2}$. The outgoing electronic current as a function of the laser intensity exhibits a slope of 4 [16] as expected from the photoelectric balance considering the work function of the gold polycrystalline sample ( $W=5.1 \mathrm{eV}$ [33]), and the photon energy $\hbar \omega_{0}=1.55 \mathrm{eV}$. As this photon energy is below the threshold $(2.3 \mathrm{eV})$ for interband transition in gold [43], SP excitation is not strongly damped and the photoelectron yield is high. Nevertheless, our measurements do not show appreciable space charge field for laser intensity lower than $5 \times 10^{10} \mathrm{~W} / \mathrm{cm}^{2}$.

As can be observed in Fig. 4, at very low laser intensity (around $1 \mathrm{GW} / \mathrm{cm}^{2}$ ), the emission is very weak and the

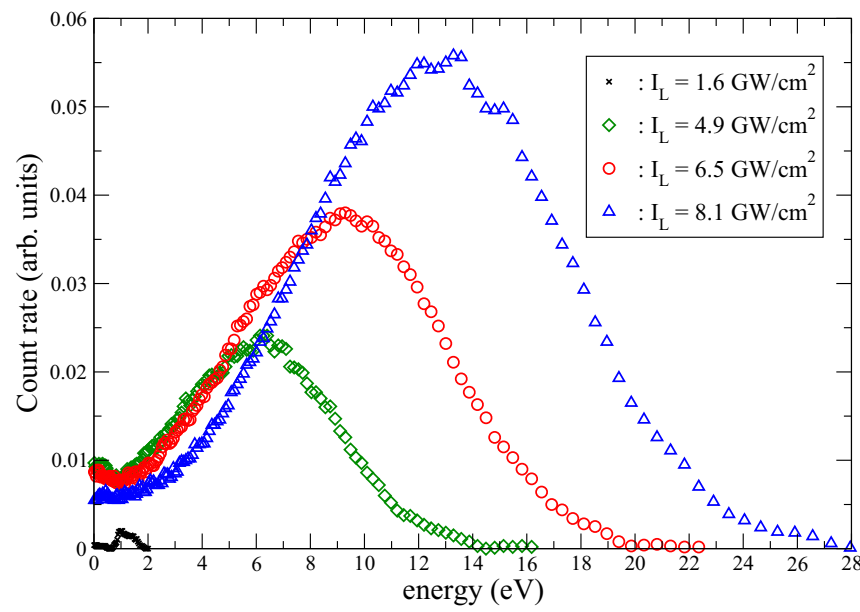

FIG. 4. (Color online) Measured photoemitted electron energy spectra of an $\mathrm{Au}$ grating irradiated with a 58 fs full width at half maximum laser pulse for four laser intensities: $\times$ : $1.6 \mathrm{GW} / \mathrm{cm}^{2}$, green diamonds: $4.9 \mathrm{GW} / \mathrm{cm}^{2}$, red circles: $6.5 \mathrm{GW} / \mathrm{cm}^{2}$, and blue triangles: $8.1 \mathrm{GW} / \mathrm{cm}^{2}$.

electron spectra mainly ranges from 0 to $1.5 \mathrm{eV}$ in agreement with the expected value $(1.1 \mathrm{eV})$ which can be deduced from the Einstein multiphoton photoelectric law. As the laser intensity increases, the number of ejected electrons strongly grows as well as the mean value and the width of the electron spectra. In that way, for the higher laser intensity considered here $\left(8.1 \mathrm{GW} / \mathrm{cm}^{2}\right)$ the mean value is around $13 \mathrm{eV}$, the width around $12 \mathrm{eV}$, and the high-energy tail extends up to $28 \mathrm{eV}$.

\section{B. Comparisons: Model-experiments}

We present in Fig. 5(a) comparison between measurements corresponding to $I_{L}=6.5 \times 10^{9} \mathrm{~W} / \mathrm{cm}^{2}$ and calculations performed by means of the approach presented in the previous

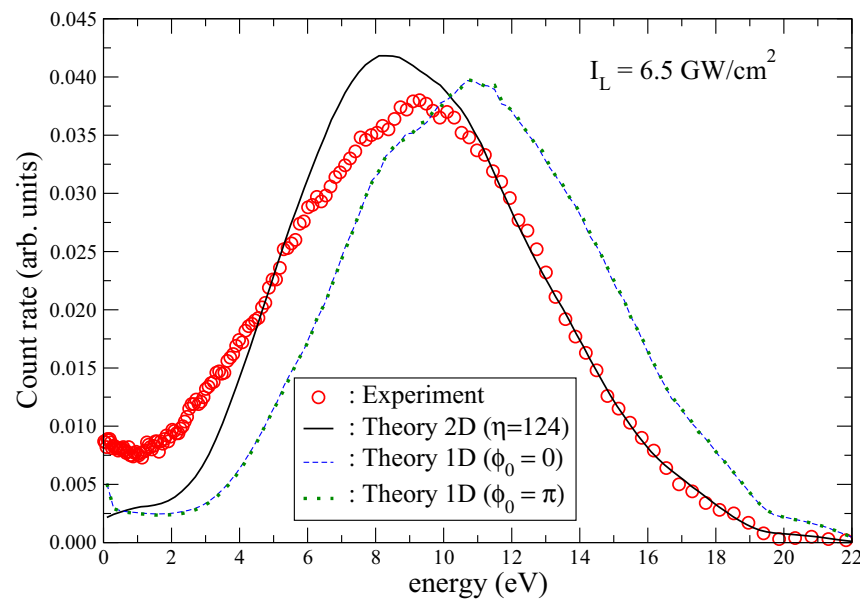

FIG. 5. (Color online) SP-enhanced emission spectrum as a function of the electron energy (in eV) for $I_{L}=6.5 \mathrm{GW} / \mathrm{cm}^{2}$. Red circles: experimental results corresponding to a 58 fs Gaussian laser pulse. Full black line: 2D theoretical spectrum (see text) obtained with $\xi_{0}^{\mathrm{SP}}=0.031$ a.u., $\tau=116 \mathrm{fs}$, and $\phi_{0}=0$. Dashed blue line: $1 \mathrm{D}$ theoretical spectrum (see text) obtained with the same parameters. Dotted green line: 1D theoretical spectrum obtained with the same parameters except the phase which is $\phi_{0}=\pi$. 
section (2D for the classical part) as well as the results of a simplified version of the calculations which consider only a 1D motion (along the direction normal to the surface) for the second (classical) part of the model. If we first focus on the comparison between the experimental data and the result of the full 2D calculation (thick full line in Fig. 5), one can see that the calculation reproduces very well the high-energy part of the experimental result which ranges between $0 \mathrm{eV}$ and $20 \mathrm{eV}$ with a maximum around $9 \mathrm{eV}$. This agreement is partially lost in the low-energy region (below $4 \mathrm{eV}$ ) where the experimental result shows more electrons than the theoretical one. This latter theoretical spectrum has been obtained by consideration of $\phi_{0}=0$ and the following SP amplitude: $\xi_{0}^{\mathrm{SP}}=\eta E_{0_{a b s}}=0.031$ a.u. [related to the primary emission results reported in Figs. 1 to 3] where $E_{0_{a b s}}$ is the amplitude of the incident laser beam corresponding to the impinging energy fraction stored in the SP wave assuming that the SP energy corresponds to the absorbed energy. As the absorption measurements provide a value of $34 \%$ for the duration considered here, the $\xi_{0}^{\mathrm{SP}}$ amplitude needed to obtain an energy location of the theoretical spectrum in agreement with the experimental one corresponds to the value $\eta=124$ for the enhancement factor. One has also to note that the area of the original theoretical spectrum has been normalized to the area of the corresponding experimental one as will also be the case for the following comparisons.

As already indicated, we also display in Fig. 5 the results of a simplified 1D calculation in which only the motion of the freed electrons on the axis perpendicular to the surface is taken into account. This amounts to neglecting the SP field component parallel to the surface and to using as an initial momentum condition only the primary energy spectrum corresponding to $\theta_{e}=90^{\circ}$ together with the temporal initial distribution described in the previous section. Obviously these $1 \mathrm{D}$ calculations are less cumbersome than the $2 \mathrm{D}$ ones either in terms of initial conditions or in terms of computation time required to integrate the classical motion equations. We report in Fig. 5 the $1 \mathrm{D}$ energy spectrum obtained for $\phi_{0}=0$ and $\xi_{0}^{\mathrm{SP}}=0.031$ a.u. (the same parameters as for the $2 \mathrm{D}$ spectrum). The 1D spectrum is very similar to the $2 \mathrm{D}$ one but it is shifted of $\sim 2.5 \mathrm{eV}$ towards high energies and then noticeably disagrees with the accurate experimental results. Hence, the effect of the SP field component parallel to the surface is to slow down the emitted electrons and the shift obtained in the $1 \mathrm{D}$ calculation relative to the $2 \mathrm{D}$ one indicates the magnitude of this effect which is important here even for the rather small SP parallel component corresponding to the case at hand. One can also see in Fig. 5 that the 1D spectrum obtained for $\phi_{0}=\pi$ is almost indistinguishable from the one corresponding to $\phi_{0}=0$. This result can be understood remembering the intermediate results related to the temporal emission regions displayed in Fig. 2. This latter figure shows that the electrons emitted in what we have called the "main" and "secondary" temporal emission regions feel the same SP field shape (although not the same amplitude) at the instant of their release independently of the phase value. The effect of the phase is then to shift these temporal emission zones within the envelope. Hence, depending on the phase, the emitted electrons experience slightly different SP field amplitudes for a surface wave composed of many cycles as is the case here ( 42 cycles), resulting in a quasi-independence of the final spectrum to the phase value. This result is important at least from a practical

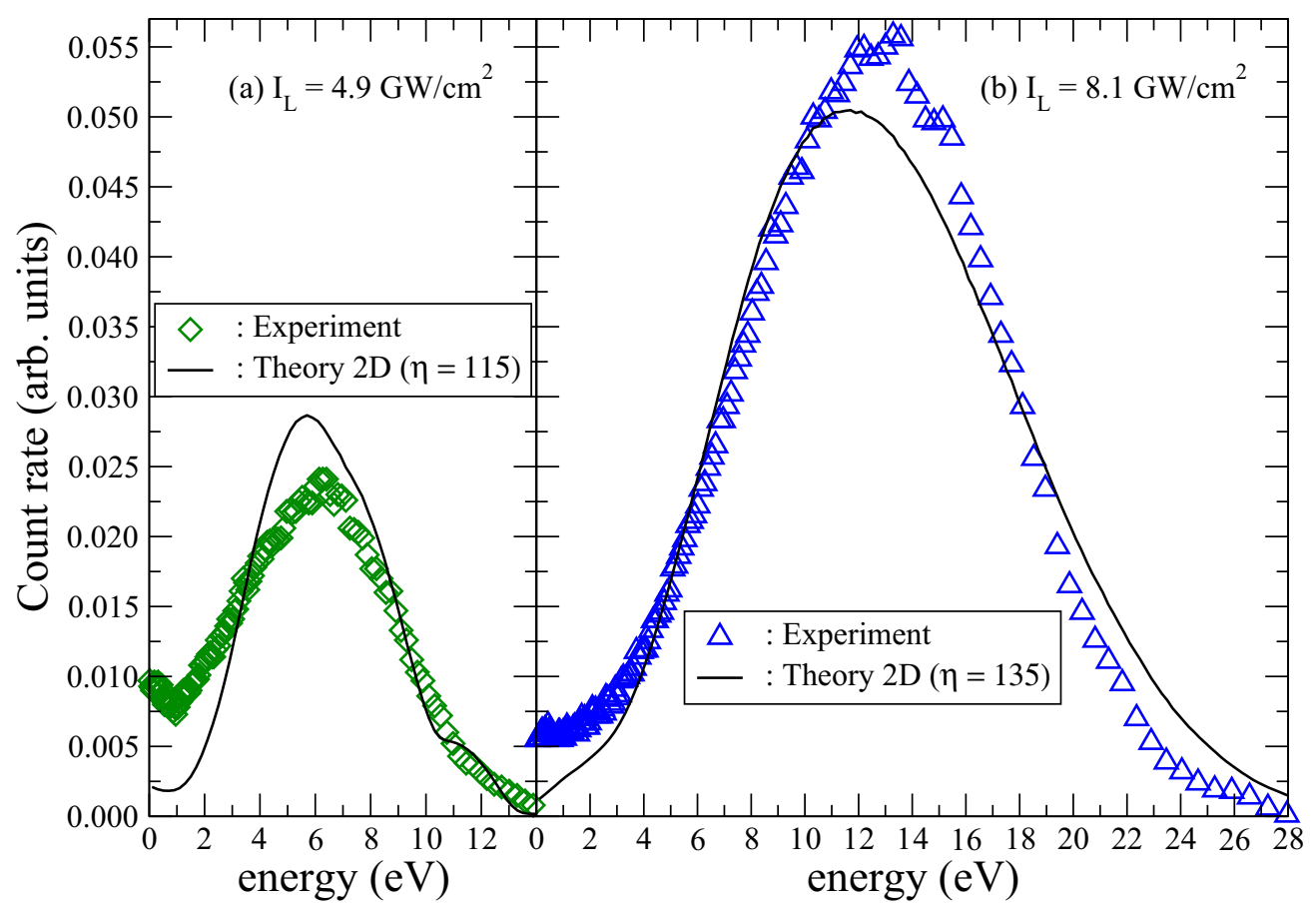

FIG. 6. (Color online) SP-enhanced emission spectrum as a function of the electron energy (in eV) for (a) $I_{L}=4.9 \mathrm{GW} / \mathrm{cm}^{2}$ and (b) $I_{L}=8.1 \mathrm{GW} / \mathrm{cm}^{2}$. Green diamonds in (a) and blue triangles in (b): Experimental results obtained with a $58 \mathrm{fs}$ Gaussian laser pulse. Thick full line in (a) and (b): $2 \mathrm{D}$ theoretical spectra obtained with $\xi_{0}^{\mathrm{SP}}=0.025$ a.u. and $\xi_{0}^{\mathrm{SP}}=0.038$ a.u., respectively. $\tau=116$ fs and $\phi_{0}=0$ in both cases. 
point of view since it shows that it is not necessary to take into account the phase in the calculations for many cycles surface waves. Obviously this would not be the case for few (or single) cycle SP fields (such as those considered in Ref. [20]) since in those cases the temporal emission zones shift due to the phase results in emitted electrons that experience very different SP strengths. Moreover, preliminary calculations on few-cycle SP waves show that the primary energy spectra also present a noticeable phase dependence.

We have reported in Fig. 6 analogous comparisons between $2 \mathrm{D}$ calculations and experimental spectra obtained for $I_{L}=$ $4.9 \times 10^{9} \mathrm{~W} / \mathrm{cm}^{2}$ [Fig. 6(a)] and $I_{L}=8.1 \times 10^{9} \mathrm{~W} / \mathrm{cm}^{2}$ [Fig. 6(b)] and the same duration as previously. The agreement between theory and experiment is quite good and similar to the one already observed in Fig. 5. The theoretical results which again show fewer low-energy electrons than the experimental ones have been obtained by consideration of $\xi_{0}^{\mathrm{SP}}=\eta E_{0_{a b s}}=$ 0.025 a.u. in case of Fig. 7(a) and of $\xi_{0}^{\mathrm{SP}}=\eta E_{0_{a b s}}=0.038$ a.u. in the case of Fig. 6(b). Taking into account the already mentioned measured value of $34 \%$ for the absorption fraction, these SP amplitudes correspond to enhancement factors of $\eta=115$ and $\eta=135$ in cases (a) and (b), respectively.

It must be noted that in the final spectra of Figs. 5 and 6 (computed or measured), the ATI peaks which are present in

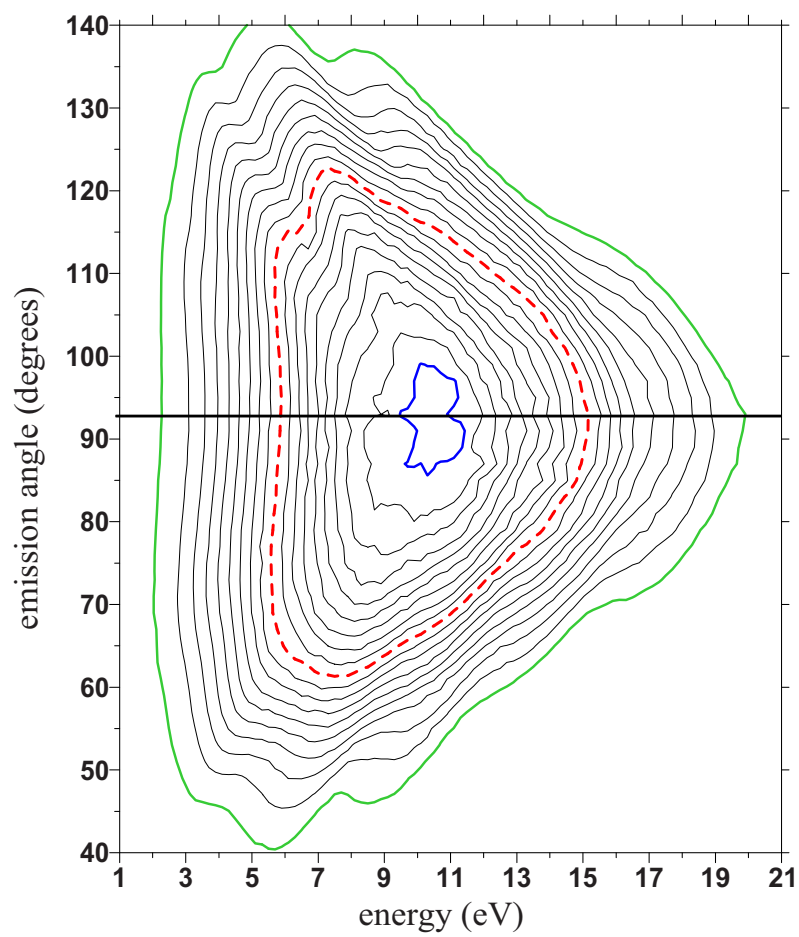

FIG. 7. (Color online) Normalized contour of the computed SPenhanced emission spectrum as a function of the electron energy (in $\mathrm{eV}$ : horizontal axis) and as a function of the emission angle (in degrees: vertical axis) for $\xi_{0}^{\mathrm{SP}}=0.031$ a.u., $\tau=116 \mathrm{fs}$, and $\phi_{0}=$ 0 . The minimum (green) level corresponds to the value 0.05 , the maximum (blue) one to the value 0.95 , and the dashed (red) line indicates the mean value 0.5 . The vertical axis can be assimilated to the surface and the laser pulse impinges with an angle of $25^{\circ}$ with respect to this axis oriented towards increasing angular values. The horizontal line approximately stands for the symmetry axis of the angular distribution. the primary energy spectra [see Fig. 1(a)] have disappeared. This washing out of the ATI structures in the final spectra is related to the second step of the process since depending on the initial conditions (energy, angle, and instant of release), the electrons can gain more or less energy during their travel outside the solid.

It is worth remembering that the amplitude of the SP field (or equivalently the enhancement factor $\eta$ ) has been used here as a free parameter. In our simulations we have observed that the energy spectra are broadened and shifted towards high energies for increasing values of the enhancement factor. In the present study, we have obtained $\eta$ values around 120 with a small variation with respect to the impinging energy. These values are approximately a factor of two smaller than the one estimated in [16] by means of the crude model used in that work. It should be also mentioned that these $\eta$ values are one order of magnitude greater than those calculated for steadystate irradiation conditions [27,44]. To our knowledge, the enhancement factor has not been obtained up to now from first principles in the case of SP excited by short laser pulses.

Finally, we report in Fig. 7 the normalized contour of the calculated energy and angular distribution of emitted electrons for the same parameters as those of Fig. 5. This distribution whose maximum is located around $9.5 \mathrm{eV}$ and $93^{\circ}$ is approximately symmetrical with respect to an axis (the horizontal line in Fig. 7) which is slightly shifted with respect to the surface normal $\left(90^{\circ}\right)$. This small shift is due to the weak SP field component parallel to the surface. The broadening of the angular distribution which is very important at small energies gets strongly reduced for increasing energies. As an example, the maximum angular width (around $60^{\circ}$ ) of the mean level (the dashed line in Fig. 7) is reached approximately at $7 \mathrm{eV}$. This angular width which is still around $50^{\circ}$ for the energy maximum gets reduced to $10^{\circ}$ near $14 \mathrm{eV}$. Comparisons with experimental angular spectra whose measurements are under study will provide more details on the SP-stimulated emission mechanism as well as on the quality of the theoretical description.

\section{CONCLUSIONS}

Within the framework of a two-step description of the surface plasmon enhanced photoemission process at metal surfaces, we have treated the first step, i.e., the primary electron ejection from the metallic conduction band, through a quantum-mechanical method. To our knowledge, this is the first time that such an approach has been implemented in the area of SP-stimulated photoelectron emission. This allows us to define accurately the initial conditions of the second (classical) step representing the motion of the freed electrons in the inhomogeneous SP field outside the solid, in terms of initial electronic momenta, angles of ejection, and instants of emission. We show that particular care must be paid in connecting both steps. Our theoretical results are in good agreement with experimental energy spectra obtained for 58 fs laser pulses impinging on a gold grating at various intensities in the $\mathrm{GW} / \mathrm{cm}^{2}$ range. These comparisons which support the two-step interpretation of the mechanism lead to almost constant values of the enhancement factor ( $\left.\eta=E_{\mathrm{SP}} / E_{0} \simeq 120\right)$ in the intensity range considered here. Calculation of the enhancement factor on theoretical grounds 
which is lacking in the literature will be the subject of a further work but is beyond the scope of the present one. Our theoretical approach has allowed us to investigate various aspects of the SP-stimulated photoemission mechanism. In particular, we have shown that the energy spectra are independent of the SP phase for SP durations of some tens of fs (or larger ones) for the wavelength considered here $(800 \mathrm{~nm})$. This would not be the case for few-cycle SP waves. Furthermore, even if the component of the SP electric field parallel to the surface is weaker than the normal one, its effect on the final energy spectra is important in such a way that it cannot be disregarded. To extend the application range of our theoretical approach the implementation of a nonperturbative quantum-mechanical method to represent the first ejection step is in progress. However, in the near future, we will apply our model in its present form which enables rather fast calculations to study the influence of wavelength or pulse duration on the SP-assisted photoemission process as well as (with some modifications) to study the stimulated photoemission of metallic nanoparticles which is a subject of growing interest.

\section{ACKNOWLEDGMENT}

M.R. thanks the technical staff of the LUCA Saclay Laser Facility for their assistance in the experiments.

\section{APPENDIX: CALCULATION OF THE MODIFIED TIME DISTRIBUTIONS}

To overcome the difficulty related to the reabsorption regions corresponding to the negative values of $d D / d t$ in Fig. 2, we split $d D / d t$ into its positive and negative parts as

$$
\frac{d D}{d t}=\frac{d D_{+}}{d t}+\frac{d D_{-}}{d t}
$$

The original time derivative distribution $d D / d t$ is then replaced by the following modified one:

$$
\begin{aligned}
\frac{d D_{M}}{d t} & =\left(\frac{\mathbf{D}}{\mathbf{D}_{+}}\right) \frac{d D_{+}}{d t}, \quad \mathbf{D}==\int_{-\tau / 2}^{+\tau / 2} \frac{d D}{d t} d t, \\
\mathbf{D}_{+} & =\int_{-\tau / 2}^{+\tau / 2} \frac{d D_{+}}{d t} d t .
\end{aligned}
$$

In that way, the reabsorption regions are eliminated from the temporal distribution and the normalization of the remaining positive part allows us to obtain a final probability (at $t=$ $+\tau / 2$ ) identical to the original one, since

$$
\int_{-\tau / 2}^{+\tau / 2} \frac{d D_{M}}{d t} d t \equiv \mathbf{D}=\int_{-\tau / 2}^{+\tau / 2} \frac{d D}{d t} d t .
$$

[1] A. Liebsch, Electronic Excitations at Metal Surfaces (Plenum Press, New York, 1997).

[2] I. I. Smolyaninov, A. V. Zayats, A. Gungor, and C. C. Davis, Phys. Rev. Lett. 88, 187402 (2002).

[3] M. Westphalen, U. Kreibig, J. Rostalski, H. Luth, and D. Meissner, Sol. Energy Mat. Sol. Cells 61, 97 (2000).

[4] W. L. Barnes, A. Dereux, and T. Ebbesen, Nature (London) 424, 824 (2003).

[5] S. Maier and H. Atwater, J. Appl. Phys. 98, 11101 (2005).

[6] S. I. Bozhevolnyi, V. S. Volkov, E. Devraux, J-Y. Laluet, and T. Ebbesen, Nature (London) 440, 508 (2006).

[7] M. I. Stockman, M. F. Kling, U. Kleineberg, and F. Krausz, Nat. Photonics 1, 539 (2007).

[8] S. Kim, J. Jin, Y. J. Kim, I. Y. Park, Y. Kim, and S. W. Kim, Nature (London) 453, 757 (2008).

[9] T. W. Ebbesen, C. Genet, and S. I. Bozhevolnyi, Phys. Today 61(5), 44 (2008).

[10] T. Tsang, T. Srinivasan-Rao, and J. Fischer, Opt. Lett. 15, 866 (1990).

[11] V. M. Shalaev, C. Douketis, T. Haslett, T. Stuckless, and M. Moskovits, Phys. Rev. B 53, 11193 (1996).

[12] P. Monchicourt, M. Raynaud, H. Saringar, and J. Kupersztych, J. Phys.: Condens. Matter 9, 5765 (1997).

[13] T. Y. Hwang, A. Y. Vorobyev, and C. Guo, Phys. Rev. B 79, 085425 (2009).

[14] A. Tredicucci, C. Gmachl, F. Capasso, A. Hutchison, D. L. Sivco, and A. Y. Cho, Appl. Phys. Lett. 76, 2164 (2000).

[15] K. Kneipp, Phys. Today 60(11), 40 (2007).

[16] J. Kupersztych, P. Monchicourt, and M. Raynaud, Phys. Rev. Lett. 86, 5180 (2001).
[17] J. Zawadzka et al., Appl. Phys. Lett. 79, 2130 (2001).

[18] S. E. Irvine, A. Dechant, and A. Y. Elezzabi, Phys. Rev. Lett. 93, 184801 (2004).

[19] G. H. Welsh, N. T. Hunt, and K. Wynne, Phys. Rev. Lett. 98, 026803 (2007).

[20] P. Rácz and P. Dombi, Phys. Rev. A 84, 063844 (2011).

[21] S. E. Irvine and A. Y. Elezzabi, Phys. Rev. A 73, 013815 (2006).

[22] P. Dombi and P. Rácz, Opt. Express 16, 2887 (2008).

[23] P. Dombi, Adv. Imaging Electron Phys. 158, 1 (2009).

[24] F. Sussmann and M. F. Kling, Phys. Rev. B 84, 121406(R) (2011).

[25] M. N. Faraggi, M. S. Gravielle, and D. M. Mitnik, Phys. Rev. A 76, 012903 (2007)

[26] P. Lu, J. Wu, H. Qi, and H. Zeng, Opt. Express 17, 4575 (2009).

[27] H. Raether, Surface Plasmon (Springer-Verlag, Berlin, 1988).

[28] D. R. Lide, Handbook of Chemistry and Physics, 84th ed. (CRC Press, New York, 2003).

[29] P. B. Johnson and R. W. Christy, Phys. Rev. B 6, 4370 (1972).

[30] D. M. Riffe, X. Y. Wang, M. C. Downer, D. L. Fisher, T. Tajima, J. L. Erskine, and R. M. More, J. Opt. Soc. Am. B 10, 1424 (1993).

[31] J. P. Girardeau-Montaut and C. Girardeau-Montaut, Phys. Rev. B 51, 13560 (1995).

[32] C. Lemell, X.-M. Tong, F. Krausz, and J. Burgdörfer, Phys. Rev. Lett. 90, 076403 (2003).

[33] A. H. Sommer, Photoemissive Materials (Krieger, Huntington, 1980).

[34] M. S. Gravielle, Phys. Rev. A 58, 4622 (1998).

[35] H. R. Reiss, Phys. Rev. A 22, 1786 (1980).

[36] G. Duchateau, E. Cormier, and R. Gayet, Phys. Rev. A 66, 023412 (2002). 
[37] J. C. Baggesen and L. B. Madsen, Phys. Rev. A 78, 032903 (2008).

[38] R. Gayet, J. Phys. B: At. Mol. Opt. Phys. 38, 3905 (2005).

[39] N. W. Ashcroft and N. D. Mermin, Solid State Physics (Saunders, Philadelphia, 1976).

[40] A. Bourgeade and G. Duchateau, Phys. Rev. E 85, 056403 (2012).
[41] M. Krüger, M. Schenk, M. Förster, and P. Hommelhoff, J. Phys. B 45, 074006 (2012).

[42] W. H. Press, S. A. Teukolsky, W. T. Vetterling, and B. P. Flannery, Numerical Recipes (Cambridge University Press, New York, 1992).

[43] B. R. Cooper, H. Ehrenreich, and H. R. Philipp, Phys. Rev. 138, A494 (1965).

[44] W. H. Weber and G. W. Ford, Opt. Lett. 6, 122 (1981). 